\title{
One Stage Posterior Minimal Laminectomy and Video-Assisted Thoracoscopic Surgery (VATS) for Removal of Thoracic Dumbbell Tumor
}

\author{
Kyoung Hyup Nam, M.D., ${ }^{1}$ Hyo Yeoung Ahn, M.D., ${ }^{2}$ Jeong Su Cho, M.D., ${ }^{2}$ Yeoung Dae Kim, M.D., ${ }^{2}$ Byung Kwan Choi, M.D., \\ In Ho Han, M.D.' \\ Department of Neurosurgery, Department of Thoracic and Cardiovascular surgery, ${ }^{2}$ Medical Research Institute, Pusan National University \\ Hosptial, Busan, Korea
}

Objective : This study was conducted to assess the surgical results of one-stage posterior minimal laminectomy and video-assisted thoracoscopic surgery (VATS) for the treatment of thoracic dumbbell tumor and to describe its precise technique. In addition, we investigated the technique's usefulness and limitations.

Methods : Seven cases of thoracic dumbbell tumor (two men and five women, mean age, 43 years) were analyzed retrospectively. Pathological findings included schwannoma in four patients, neurofibroma in two patients, and hemangioma in one patient. The location of tumors varied from T2/3 to T12/L1. Dumbbell tumors were resected by one-stage operation using posterior laminectomy followed by VATS without instrumentation. Clinical data were reviewed.

Results : The mean follow-up period was 25 months (range, 3-58 months), and the operative time ranged from 255 to 385 min (mean, $331 \mathrm{~min}$ ), with estimated blood loss ranging from 110 to $930 \mathrm{~mL}$ (mean, $348 \mathrm{~mL}$ ). The tumor was completely resected without instrumentation and postoperative instability in all cases. Postoperative complications included atelectasis and facial anhydrosis in one case each.

Conclusion : One-stage posterior minimal laminectomy and VATS may be a safe and less invasive technique for removal of thoracic dumbbell tumor without instability. This method has the advantage of early ambulation and rapid recovery because it reduces blood loss and postoperative pain.

Key Words : Thoracic dumbbell tumor · Laminectomy · VATS · One-stage operation.

\section{INTRODUCTION}

Surgical resection of thoracic dumbbell tumors is occasionally challenging because it necessitates surgical approach to three compartments (intraspinal, foraminal, and mediastinal).
Although several surgical approaches such as single or multiple staged, combined laminectomy and open thoracotomy, or single-staged posterior wide laminectomy with instrumentation have been introduced, there has been no definite standard technique until now ${ }^{1,2,9,11}$. Currently, combined posterior

- Received : September 26, 2016 •Revised : December 9, 2016 •Accepted : December 23, 2016

- Address for reprints : In Ho Han, M.D., Ph.D.

Department of Neurosurgery, Pusan National University Hospital, 179 Gudeok-ro, Seo-gu, Busan 49241, Korea

Tel : +82-51-240-7257, Fax : +82-51-244-0282, E-mail : farlateral@hanmail.net

This is an Open Access article distributed under the terms of the Creative Commons Attribution Non-Commercial License (http://creativecommons.org/licenses/by-nc/4.0) which permits unrestricted non-commercial use, distribution, and reproduction in any medium, provided the original work is properly cited. 
laminectomy and video-assisted thoracoscopic surgery (VATS) has been introduced as an alternative method to remove thoracic dumbbell tumor in several case reports ${ }^{5,7,10,12}$. Here, we describe a one-stage posterior minimal laminectomy and VATS technique as a less invasive technique to remove thoracic dumbbell tumor and discuss the technique's usefulness and limitations.

\section{MATERIASL AND METHODS}

\section{Patients}

Between 2009 and 2015, one-stage posterior minimal laminectomy and VATS was performed to remove thoracic dumbbell tumor in seven patients (two men and five women). The mean age of the patients was 43 years (ranged from 30 to 57), and the level of tumors varied from T2/3 to T12/L1 (Table 1).

\section{Surgical procedures}

The first step in posterior hemilaminectomy is a posterior midline incision of $5 \mathrm{~cm}$ in the prone position. Unilateral paravertebral muscle dissection is then conducted to expose the lamina and facet joint. Next, hemilaminectomy is performed preserving the minimal lateral half facet joint with a high speed drill. After the thecal sac and intraspinal tumor mass are exposed, the involved nerve root is identified. The intraspinal mass is removed by a piecemeal technique and a small amount of tumor material is sent for frozen biopsy, after which pathological findings are identified. When the thecal sac is decompressed and the involved nerve root is resected, the normal proximal nerve root remains. In most cases, tho- racic dumbbell tumor does not invade the intradural space, and the normal proximal part of the nerve root can be identified. After meticulous bleeding control, the wound is closed.

After the patient's position was changed to lateral decubitus, an initial $1.1 \mathrm{~cm}$ incision was made in the 7th-8th intercostal space (ICS) on the mid-axillary line. Thoracoscope $\left(30^{\circ}\right.$ direction and $10 \mathrm{~mm}$ diameter) was introduced to inspect the pleura. The other ports were inserted through the 3rd-4th ICS in the anterior axillary line and 6th-7th ICS in the posterior axillary line to make triangular configuration. After the thoracoscope was reinserted through the port in the 3rd-4th ICS in anterior axillary line, the instruments were reemployed through the other ports for grasping and dissection of tissues. After the thoracoscope was reinserted through the port in the 3rd-4th ICS in anterior axillary line, the instruments were reemployed through the other ports for grasping and dissection of tissues.

After division of the parietal pleura around the tumor, the tumor was usually dissected from the bottom, preventing injury of the intercostal artery and vein. If the intercostal artery and vein were drained to the tumor or interrupted the dissection, they were divided using endo clips. Counter traction of the tumor enabled its complete dissection. The tumor was extracted through the port in the 3rd-4th ICS in the anterior axillary line using an endobag (Fig. 1).

The tumor surface and involved nerve root, which were resected by intraspinal approach, were identified to confirm total tumor resection. The Jackson-Pratt catheter was inserted after meticulous bleeding control (Fig. 2).

Table 1. Characteristics of patients who underwent one-stage posterior laminectomy and VATS for thoracic dumbbell tumor

\begin{tabular}{|c|c|c|c|c|c|c|c|c|}
\hline Case & Age/sex & Diagnosis & Level & $\begin{array}{c}\text { Eden } \\
\text { classification }\end{array}$ & $\begin{array}{l}\text { OP time } \\
\text { (min) }\end{array}$ & $\begin{array}{c}\text { EBL } \\
(\mathrm{mL})\end{array}$ & $\begin{array}{l}\text { Ambulation } \\
\text { (POD) }\end{array}$ & Complication \\
\hline 1 & $30 / M$ & Schwannoma & $\mathrm{T} 2 / 3$ & Type 4 & 375 & 300 & 1 & Anhydrosis \\
\hline 2 & $38 / \mathrm{M}$ & Schwannoma & $\mathrm{T} 10 / 11$ & Type 3 & 380 & 400 & 1 & \\
\hline 3 & $46 / F$ & Neurofibroma & T8/9 & Type 4 & 260 & 150 & 3 & Atelectasis \\
\hline 4 & $57 / F$ & Hemangioma & $\mathrm{T} 2 / 3$ & Type 3 & 385 & 930 & 1 & \\
\hline 5 & $56 / F$ & Schwannoma & $\mathrm{T} 4 / 5$ & Type 3 & 255 & 110 & 1 & \\
\hline 6 & $35 / F$ & Neurofibroma & $\mathrm{T} 3 / 4$ & Type 3 & 370 & 350 & 1 & \\
\hline 7 & $37 / F$ & Schwannoma & $\mathrm{T} 12 / \mathrm{L} 1$ & Type 4 & 385 & 200 & 1 & \\
\hline
\end{tabular}

VATS : video-assisted thoracoscopic surgery, OP : operation, EBL : essential blood loss, POD : post-operative day 


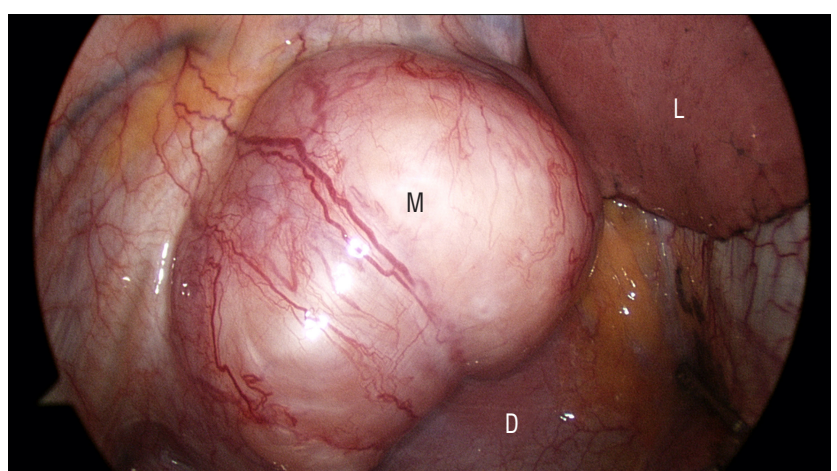

Fig. 1. Intraoperative thoracoscopic finding shows a round mediastinal mass extruding over a diaphragm at T12/L1. M : mass, D : diaphragm, L : lung.

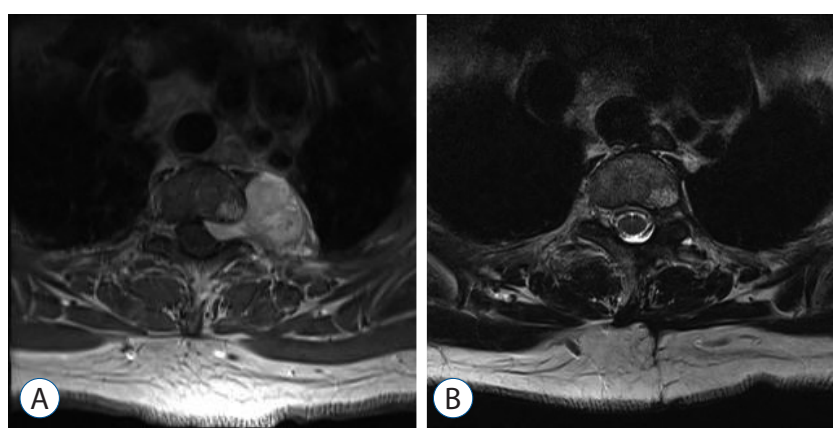

Fig. 3. A : Preoperative gadolinium-enhanced MR image showing a wellenhanced dumbbell shaped tumor with a neuroforaminal extension in the left paravertebral space and posterior mediastinum at T2/3. B : Postoperative T2-weighted MR image shows no remnant tumor and partial laminectomy state reserving lateral facet. MR : magnetic resonance.
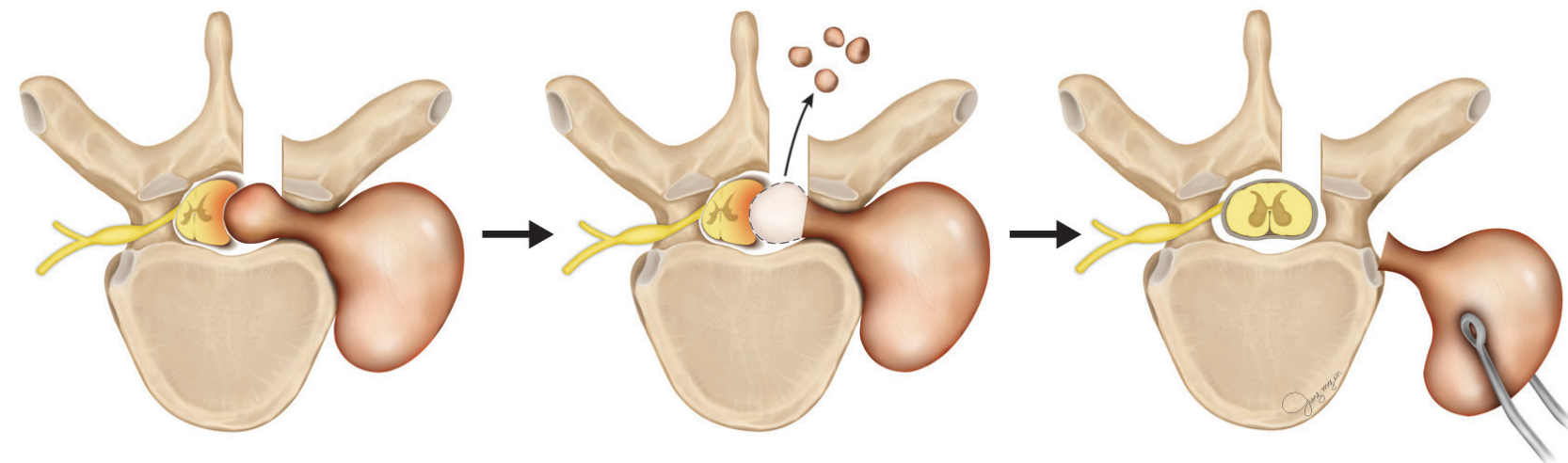

Fig. 2. Schematic illustration of one stage posterior minimal laminectomy and VATS for removal of thoracic dumbbell tumor. VATS : video-assisted thoracoscopic surgery.

\section{RESULTS}

The mean operative time was 331 minutes (range 255 to 385 min), with estimated blood loss (EBL) of 110 to $930 \mathrm{~mL}$ (mean, $348 \mathrm{~mL}$ ). In six patients, the EBL was less than 400 $\mathrm{mL}$. In one patient, the EBL was $930 \mathrm{~mL}$ because the pathological finding was unexpectedly hemangioma. Complete tumor resection was accomplished without instrumentation and postoperative instability in all patients. Postoperative complications included atelectasis and facial anhydrosis in one patient each. One day after operation, the patient with atelectasis underwent bronchoscopy and atelectasis was immediately improved. In a patient with a dumbbell tumor at T2/3, facial anhidrosis occurred on the affected side due to sympathetic trunk injury, and this conditions still persisted after two years. All patients except for one with atelectasis were ambulatory one day after operation. Final pathologic findings included schwannoma in three patients, neurofibroma in three, and hemangioma in one (Table 1). The postoperative follow-up period was 3 to 58 months (mean : 18.5 months). There was no tumor recurrence, spinal instability or pain in any patients upon final follow-up.

\section{ILLUSTRATE CASE PRESENTATIONS}

A 55-year-old female (Case 4) admitted to our hospital with left thoracic radiating pain. MRI revealed a dumbbell shaped tumor with a neuroforaminal extension in the left paravertebral space and posterior mediastinum at T2/3 (Fig. 3A). We decided to perform one stage minimal posterior hemilaminectomy and VATS. During posterior laminectomy, the tumor was 
found to not originate from the nerve root and showed unexpected bleeding during intraspinal piecemeal removal. After complete removal of the intraspinal tumor, the exiting T2 nerve root was sacrificed for tumor extraction during VATS. After posterior approach, VATS was performed through three ports and the mediastinal mass was found to be ovoid with a diameter of $3 \mathrm{~cm}$ diameter in thoracoscopic view and appeared to be hypervascular. The tumor was doubled with the sympathetic chain and therefore, the proximal and distal chains of tumor were divided with clips. The mediastinal portion of the mass was removed en bloc by circumferential dissection. The final diagnosis of the dumbbell tumor was hemangioma. There were no complications and only minimal hypesthesia at the right $\mathrm{T} 2$ dermatome (Fig. 3B).

\section{DISCUSSION}

There have been several efforts to treat thoracic dumbbell tumors. In general, a single stage posterior approach including partial laminectomy and unilateral facetectomy has been performed ${ }^{1,111}$. However, more invasive procedures are required for tumors with large intra-thoracic components, including wide incision and resection of adjacent structures (rib, transverse process and pedicle). For stabilizing the spine, spinal reconstruction with fusion instrument should be used ${ }^{1}$.

Based on Eden classification, dumbbell tumors are classified into 4 types, as follows : Type 1 is intradural and extradural; type 2 is intradural, extradural, foraminal, and paravertebral; type 3 is extradural, foraminal, and paravertebral; and Type 4 is foraminal and paravertebral ${ }^{6}$. Type 3 dumbbell tumors are usual in the thoracic spine ${ }^{8}$. In this study, all dumbbell tumors were classified into type 3 or 4 . Thus, durotomy for removal of the mass was not essential in our cases.

First, partial laminectomy is performed to remove the intraspinal component of dumbbell tumors, after which open thoracotomy is conducted to remove the intrathoracic component. However, open thoracotomy requires division of one or more major muscles of the chest wall, including the latissimus dorsi, pectoralis, or serratus muscles with spreading of the ribs. To avoid invasive thoracotomy, VATS was introduced in the early $1990 \mathrm{~s}^{3,4)}$. Currently, VATS is applied widely in various thoracic surgeries including resection of lung or mediastinal masses. Thoracoscopic incision is less invasive, sparing di- vision of major muscles, which results in less postoperative pain, early recovery and lower morbidity ${ }^{3,4}$. Due to the feasibility of VATS, combined laminectomy and VATS have been used to remove thoracic dumbbell tumors ${ }^{5,10,12)}$. However, the combined approach for thoracic dumbbell tumor has only been reported in case reports or technical notes due to rarity of thoracic dumbbell tumor. Although our series only included seven cases, a combined approach using VATS was applied in the largest number of patients. In our cases, a combined approach was successfully performed from T2/3 to T12/L1. Considering the possible approach of VATS to T1/2, the combined approach could be applied for dumbbell tumors of all levels. However, sympathetic chain injury can occur during removal of upper thoracic tumors, and meticulous care is required.

The best advantage of combined approach is early ambulation of patients due to less invasiveness. In our cases, all patients ambulated the day after operation, except for one with atelectasis. In addition, transfusion was not required because of lesser intraoperative bleeding below $400 \mathrm{~mL}$. However, a combined approach may be difficult because intraoperative bleeding can obscure the operative field and VATS enables only limited bleeding control in highly vascular tumors. In our case of hemangioma, blood loss of $500 \mathrm{~mL}$ occurred during piecemeal removal of the intraspinal tumor. Therefore, we recommend that preoperative tumor embolization be considered in a combined approach for highly vascular dumbbell tumors.

There is still debate regarding whether this combined approach is really less invasive. The operation time of the combined approach is 4 to 6 hours, which is similar to that of the single posterior approach with instrumentation. Risks of postoperative pulmonary complications such as atelectasis or pleural effusion always exist. Nevertheless, our opinion is that the combined approach is less invasive than a single posterior approach because it requires no spinal instrumentation, and results in less intraoperative bleeding, less postoperative pain, and early ambulation. Therefore, a combined approach is a good alternative method for removal of thoracic dumbbell tumors.

\section{CONCLUSION}

One-stage posterior minimal laminectomy and VATS are a 
safe and less invasive technique for removal of thoracic dumbbell tumor without instrumentation and instability. The approach could be applied for all surgical levels. This method can reduce intraoperative bleeding and postoperative pain, resulting in early ambulation and recovery. In highly vascular tumors, preoperative embolization should be considered to reduce intraoperative bleeding.

\section{References}

1. Ando K, Imagama S, Ito Z, Tauchi R, Muramoto A, Matsui H, et al. : Removal of thoracic dumbbell tumors through a single-stage posterior approach : its usefulness and limitations. J Orthop Sci 18 : 380-387, 2013

2. Ando K, Imagama S, Wakao N, Hirano K, Tauchi R, Muramoto A, et al. : Single-stage removal of thoracic dumbbell tumors from a posterior approach only with costotransversectomy. Yonsei Med J 53 : 611-617, 2012

3. Hurley JP, McCarthy J, Wood AE : Retrospective analysis of the utility of video-assisted thoracic surgery in 100 consecutive procedures. Eur J Cardiothorac Surg 8 : 589-592, 1994

4. Jeon JH, Kang CH, Kim HS, Seong YW, Park IK, Kim YT, et al. : Videoassisted thoracoscopic lobectomy in non-small-cell lung cancer patients with chronic obstructive pulmonary disease is associated with lower pulmonary complications than open lobectomy : a propensity scorematched analysis. Eur J Cardiothorac Surg 45 : 640-645, 2014

5. Jeong WJ, Choi I, Seong HY, Roh SW : Thoracic extradural cavernous hemangioma mimicking a dumbbell-shaped tumor. J Korean Neurosurg Soc $58:$ 72-75, 2015

6. Eden K : The dumbbell tumours of the spine. Br J Surg 28 : 549-570, 1941

7. Konno S, Yabuki S, Kinoshita T, Kikuchi S : Combined laminectomy and thoracoscopic resection of dumbbell-type thoracic cord tumor. Spine (Phila Pa 1976) 26 : E130-E134, 2001

8. Ozawa H, Kokubun S, Aizawa T, Hoshikawa T, Kawahara C : Spinal dumbbell tumors : an analysis of a series of 118 cases. J Neurosurg Spine 7 : 587-593, 2007

9. Payer M, Radovanovic I, Jost $G$ : Resection of thoracic dumbbell neurinomas : single postero-lateral approach or combined posterior and transthoracic approach? J Clin Neurosci 13 : 690-693, 2006

10. Shiraishi T, Hida S, Isayama T, Yoneda S, Kawahara K, Shirakusa T : A combined thoracoscopic and posterior-spinal approach for "dumbbell" neurofibroma minimizes the anatomical destruction of the vertebrae : report of a case. Surg Today 32 : 155-158, 2002

11. Thorat JD, Rajendra T, Thirugnanam A, Ng IH : Single-stage posterior midline approach for dumbbell tumors of the thoracic spine, with intraoperative CT guidance. Surg Neurol Int 2 : 31, 2011

12. Vallieres $E$, Findlay JM, Fraser RE : Combined microneurosurgical and thoracoscopic removal of neurogenic dumbbell tumors. Ann Thorac Surg 59 : 469-472, 1995 